\title{
OPERATIONAL EXPERIENCE WITH X-RAY BEAM POSITION MONITORS AT THE ADVANCED PHOTON SOURCE*
}

\author{
O. Singh ${ }^{\dagger}$ and G. Decker \\ Advanced Photon Source, Argonne National Laboratory, Argonne, IL
}

\begin{abstract}
Photoemission-based $\mathrm{x}$-ray beam position monitors (XBPMs) have been employed in each of the bending magnet (BM) as well as insertion device (ID) beamline front-ends in the Advanced Photon Source storage ring. The position data derived from the BM XBPM blades have been achieved at the few-micron rms level. Insertion device XBPM-based position data have historically been plagued with problems resulting from background stray radiation impinging on the blades. A method that involves introducing chicanes into the lattice at both sides of the ID was proposed and implemented in APS sector 34 in December 1998, resulting in the elimination of most of the unwanted radiation. The effects of the remaining background radiation were further reduced by a background subtraction procedure. New DSP-based data acquisition and control hardware has been installed that allows the smooth integration of XBPM data with the existing storage ring rf-BPM system. XBPM-based orbit feedback, for a fixed ID gap, has resulted in vertical pointing stability of $0.2 \mu \mathrm{rad}$ rms over a $24-\mathrm{h}$ time period. Because of these good results, chicanes have been introduced for six more beamlines and several more are planned in the future. Operational experience with these beamlines is presented and progress on methodologies for reducing gap-dependent orbit motions is discussed.
\end{abstract}

\section{INTRODUCTION}

Position data from $\mathrm{x}$-ray beam position monitors (XBPMs) at the Advanced Photon Source (APS) have been available to users for alignment purposes for several years [1], but not for continuous orbit control. With the new data acquisition and control hardware [2,3], XBPM-based orbit control is now possible and commissioning is in progress. Several bending magnet (BM) beamlines are now routinely employing XBPMbased orbit feedback, with others to be completed by the end of calender year 2001. The study is in progress for employing insertion device (ID) XBPMs in orbit feedback where chicanes have been introduced.

The orbit control at Advanced Photon Source [4][5] employs workstation based (DC) orbit correction [6] as well as digital signal processor(DSP)-based real-time orbit feedback [7], separated in frequency bands. Presently, only a workstation-based orbit correction

\footnotetext{
* Work supported by the U.S. Department of Energy, Office of Basic Energy Sciences, under Contract No. W-31-109-ENG-38.

‘singh@aps.anl.gov
}

system employs XBPMs to correct the orbit to $\sim 0.1 \mathrm{~Hz}$. This system implements XBPMs in a local bump configuration, which is achieved by the addition of correctors straddling the source and by assigning XBPMs higher weights than radio frequency BPMs $($ RFBPMs) $($ XBPM weight $=5$; RFBPM weight $=1$ ), integrated into a global singular valve decomposition (SVD) algorithm.

Figure 1 shows a schematic diagram indicating the locations of both types of BPMs. The BM XBPM uses
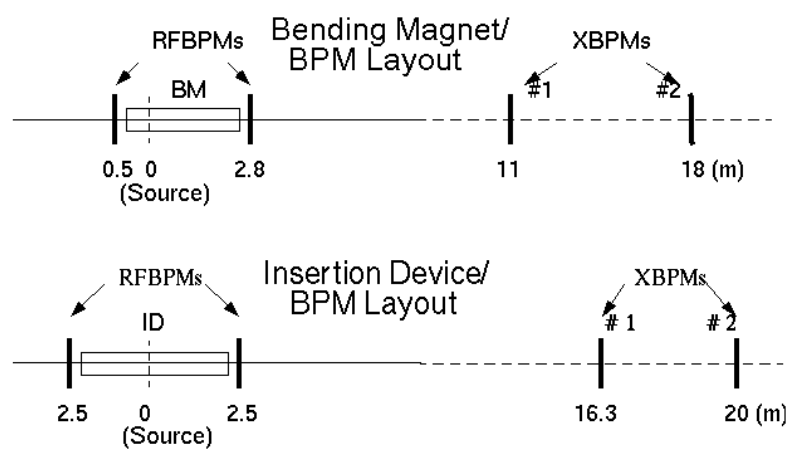

Figure 1: BPM layout for BM and ID.

a pair of vertical blades, with downstream XBPM blades slightly off-centered horizontally to avoid shadow effect. The ID XBPM uses two pair of blades. For the standard layout, Fig. 2 shows the blades' geometries for the upstream and the downstream XBPMs.

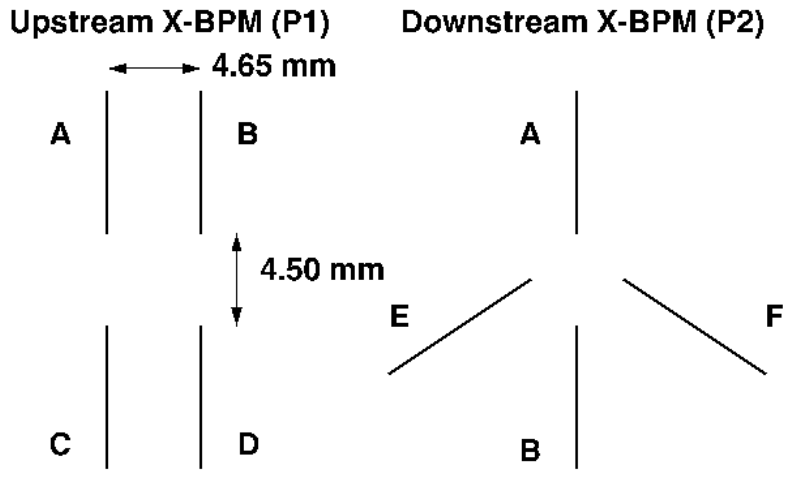

Figure 2: APS ID blades' geometries.

The positions for this XBPM's configuration (ID) are computed as follows: 


$$
\begin{aligned}
& \mathrm{P} 1 \mathrm{y}=\mathrm{K} 1_{\mathrm{y}}(\mathrm{A}+\mathrm{B}-\mathrm{C}-\mathrm{D}) /(\mathrm{A}+\mathrm{B}+\mathrm{C}+\mathrm{D}) \\
& \mathrm{P} 1 \mathrm{x}=\mathrm{K} 1_{\mathrm{x}}(\mathrm{A}+\mathrm{C}-\mathrm{B}-\mathrm{D}) /(\mathrm{A}+\mathrm{B}+\mathrm{C}+\mathrm{D}) \\
& \mathrm{P} 2 \mathrm{y}=\mathrm{K} 2_{\mathrm{y}}(\mathrm{A}-\mathrm{B}) /(\mathrm{A}+\mathrm{B}) \\
& \mathrm{P} 2 \mathrm{x}=\mathrm{K} 2_{\mathrm{x}}(\mathrm{E}-\mathrm{F}) /(\mathrm{E}+\mathrm{F})
\end{aligned}
$$

Due to the unique layout of $\mathrm{P} 2$, blades $\mathrm{E} / \mathrm{F}$ get most of the remaining stray radiation, making the $\mathrm{P} 2 \mathrm{x}$ measurement most susceptible to background radiation. Two of approximately 20 ID beamlines employ P2 blade geometry similar to P1's, reducing the effect of background radiation.

All XBPM assemblies are also equipped with translation stages with computer-controlled stepper motors. These stages are driven precisely down to micron level with repeatable accuracy, and thus, are also utilized to calibrate the XBPM electronics. Once XBPMs are calibrated, XBPM position data are used to cross-calibrate RFBPMs that straddle the source point, using local bumps. Calibration results are presented later in this paper.

\section{EXPERIENCE WITH BENDING MAGNET XBPMS}

BM XBPM-based position data have been reliable and repeatable, and have been in use for several years to align a user beam after either a long shutdown or a brief maintenance period. New DSP-based XBPM data acquisition system and control hardware allows the integration of XBPMs with existing RFBPMs into a unified orbit control configuration. For the RFBPMs, a unique "despiking" algorithm [6]] is used to take care of bad BPM readbacks having nonphysical changes. For XBPMs, at least for now, we plan to use one XBPM for orbit feedback while we use other XBPM for monitoring as a watchdog. When any one XBPM reading falls out of a certain range (nominally $+/-0.5$ $\mathrm{mm})$, the orbit control is interrupted and the operator is alerted. A photon shutter (PS1) located just upstream of XBPM P2 ensures proper feedback operation; the orbit-control algorithm also monitors its status.

Orbit drifts for five 12-h fills, as observed by XBPMs in BM14 and BM33, are shown in Fig. 3.

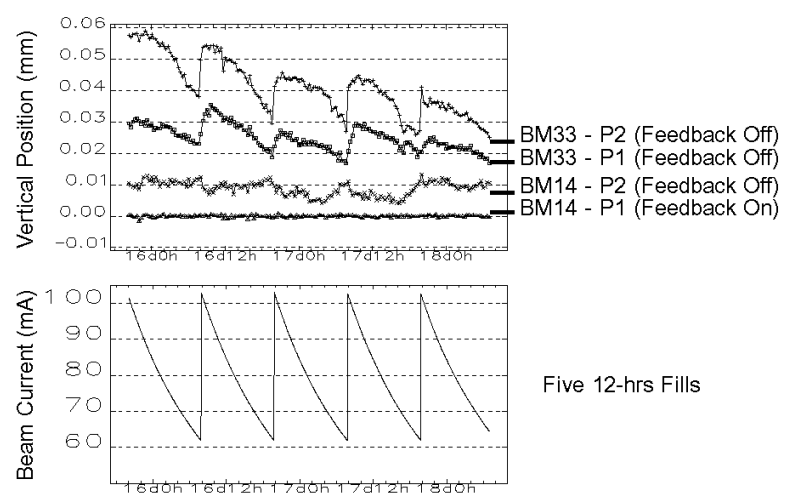

Figure 3: Two-bending-magnet XBPM data with BM14 XBPM P1 feedback ON.
During this period, only BM14 XBP P1 is included in orbit control. The BM33 XBPMs, which are not included in orbit control, show orbit drifts from 10 to $20 \mu \mathrm{m}$ during 12-h fills. These XBPMs also indicate a total drift "walk" of 10 to $20 \mu \mathrm{m}$ for the entire five fill periods. BM14 XBPM P2's orbit drift and orbit "walk" have been reduced to about $5 \mu \mathrm{m}$ p-p, with almost no observable motion on BM14 XBPM P1, which has been forced to zero by the algorithm. For a user at $30 \mathrm{~m}$ away from the dipole source, 5- $\mu \mathrm{m}$ p-p converts to a vertical pointing stability of $0.1 \mu \mathrm{rad} \mathrm{rms}$.

\section{EXPERIENCE WITH INSERTION DEVICE XBPMS}

The position measurement with ID XBPMs has been a challenge at almost all synchrotron facilities [8][9], mainly due to the background stray radiation impinging on the blades. For large gaps especially, the ID photon flux is quite small in proportion to the stray radiation. Further exacerbating the problem is the fact that the stray radiation sources are variable, for example when local steering is performed at an adjacent BM source point.

\subsection{Lattice Modification}

A method to eliminate background radiation has been implemented for several sectors at APS by the introduction of chicanes into the lattice at both sides of the ID, thus directing unwanted stray radiations away from the blades [10]. Even though the lattice modification diverts all existing sources of stray radiation away from the blades, it does introduce two 1-mrad horizontal corrector radiation sources. The net effect is that the lattice modification has reduced the amount of background radiation impinging on the blades (sum of all four blades) by a factor of more than eleven [11] for the upstream XBPM (P1), but only by a factor of two for the downstream XBPM (P2). However, with a tight control over the orbit near the insertion device, it is expected that 1-mrad corrector radiation should be predictable, and thus can be subtracted out successfully by a background subtraction method, described below. Another approach to reduce background radiation on $\mathrm{P} 2-\mathrm{E} / \mathrm{F}$ blades is to change to the P1-type geometry. As mentioned previously, this geometry has been implemented at two beamlines, resulting in a reduction of the background radiation level in $\mathrm{P} 2$ to a level that is comparable to that in P1. An upgrade to all XBPM P2 configurations is being considered.

\subsection{Background Subtraction}

The effect of background radiation is reduced by a "background subtraction" procedure, where the background radiation signal is subtracted from the blade signals prior to computing the position. This technique is especially useful when XBPM position 
measurements are made as the gap varies. When the electron orbit external to the ID is kept fixed as gap varies, it is a fair assumption that the background radiation levels are not changing and thus can be subtracted out accurately. Under these conditions with background subtraction, the XBPM position changes during a gap variation are strictly the effects of the ID.

Figure 4 shows a medm screen with all eight blade's signals with several columns at various computing levels. The first column is the measured average blade data, which then is normalized to the beam current and inserted into the second column. The normalized data is adjusted by subtracting "normalized offset data" (fifth column) and the resulting adjusted data are then used to compute position. The data for offset column is determined prior to the operation with several iterations of gap closing/opening. To start, the gap is closed to its minimum so that the position errors due to background radiations are small. The translation stages are adjusted to zero out each position. We have chosen "zero" to be our operating point, but it could be any other point nearby in the linear range. Now, the gap is opened to a large value (usually $>60 \mathrm{~mm}$ ) and the normalized blade signal levels are transferred to the offset column. The process is repeated at least one more time, to verify whether an additional iteration is required. The results of background subtraction are presented later.

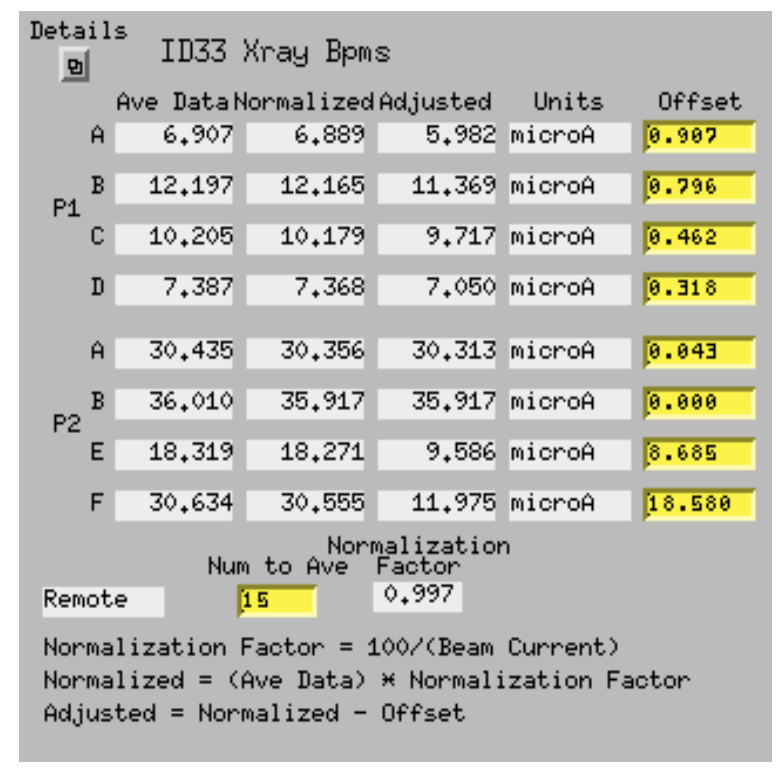

Figure 4: Background subtraction medm display.

\subsection{Results at Fixed Gap}

The XBPM-based orbit feedback results for ID32, where lattice modification has been made, are shown in Fig. 5. This is a $16-\mathrm{h}$ operational run with $27.5-\mathrm{mm}$ fixed gap where XBPMs are included in orbit feedback only during the first half period. For this test, we chose, arbitrarily, to include both XBPMs in the vertical plane, but only one downstream XBPM in the horizontal plane. Note that the flatness of the two vertical traces during the period when XBPMs were included merely indicates that the correction algorithm is working. Nevertheless, because the vertical openloop readbacks are well correlated at the few-micron level, it is likely that a significant improvement has been achieved by including these XBPMs in the algorithm. Some kind of collaboration will be required between the experimenters at the beamline and the APS staff to determine the true beam stability level, for example, by measuring the intensity variation in an ion chamber detector located downstream of both XBPMs.

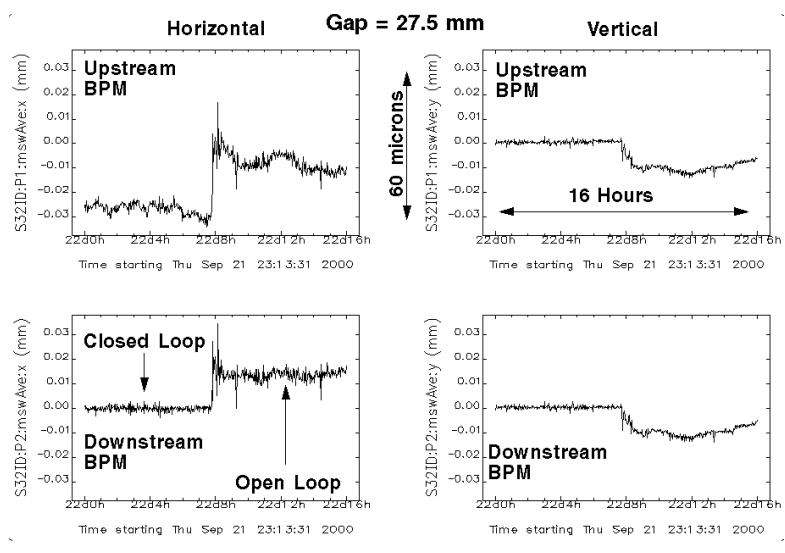

Figure 5: Sector 32 ID XBPM data with and without including XBPMs in the orbit feedback.

\subsection{Insertion Device Gap Change Effects}

The XBPM position measurements are affected in several ways as the gap varies:

- Electron orbit changes outside the ID due to ID steering effects.

- Electron orbit changes internal to the IDs also from ID steering.

- Changes in XBPM gains (sensitivities), probably due to change in undulator radiation spectrum and/or variation in the XBPM blade's response due to thermal effects or fabrication errors from unit to unit $[1,8]$.

The changes in the electron orbit as it enters and leaves the ID is minimized, at least to first order, by feedforward method, where two straddling correctors are adjusted as the gap varies. For now, the workstation will be employed to perform feedforward correction where the update rate is limited to few seconds. In the near future, this correction will be made at the level of the local processors, which have quick access to the local correctors and gap-level information; it is expected that the correction will then be applied at a rate of tens of Hz. The residual orbit is further reduced by the global orbit correction. The corrector settings, used by the feedforward algorithm, are determined by using an orbit control configuration 
with only these two correctors and all possible RFBPMs.

Correction for the orbit changes inside the insertion device is much more difficult; it is not clear what the best strategy should be. The changes in XBPM position measurement are believed to be some combination of (1) orbit changes within ID, (2) varying undulator radiation geometrical patterns [8], and (3) varying XBPM blade's response due to thermal effects and/or fabrication errors from unit to unit. We plan to study these effects in near future.

Figure 6 shows the results from an experiment where XBPM position measurements (with background subtraction) are made with translation stages centered (traces C) and then displaced by $200 \mu \mathrm{m}$ (traces D), while the electron trajectory is fixed using RFBPMs as the gap varies. The corresponding traces C' and D' are the position measurement with no background correction applied. As shown, the position measurement error, caused by the presence of background radiations, increases as the gap increases, which is attributed to the decrease in the undulator radiation level compared with the background radiation.

In this experiment, three gap scans were taken. The first scan was done with all XBPMs centered with the beam. In the second and third scans, the translation stages were moved $200 \mu \mathrm{m}$, first in the horizontal direction and then in the vertical direction. It is believed that during all three scans the residual photon beam position variation should repeat as the gap varies, and so any difference in measurement would mostly be due to the effective XBPM gains (sensitivities) variation. In Fig. 6, it is observed that for both XBPMs, horizontal gains increases and vertical gain decreases as the gap increases.
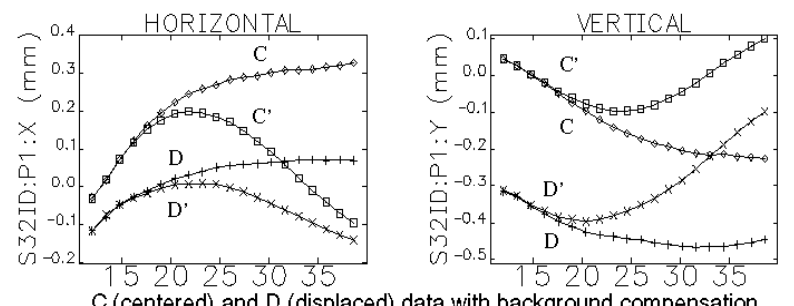
(centered) and D (displaced) data with background compensation
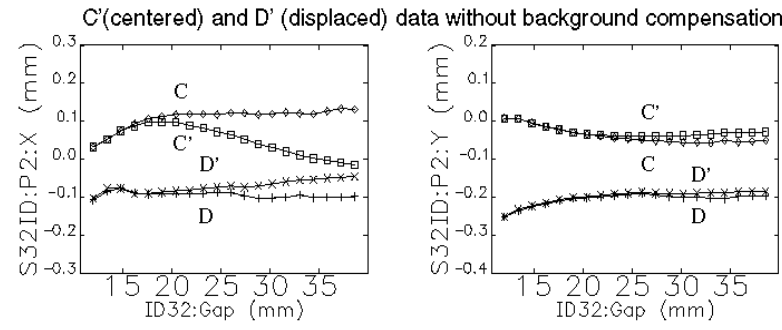

Figure 6: Gap change effects on XBPM gain (sensitivity) with and without background subtraction when translation stage was (1) centered (C \& C') and (2) displaced (D \& D') by 200 microns.
This manifests itself in the changing relative separation between the $\mathrm{C}$ and $\mathrm{D}$ curves as the gap is varied. The gain variation data will be incorporated in XBPM electronics in the future.

\section{XBPM AND RFBPM CALIBRATION}

The first step is to calibrate XBPM electronics by moving the XBPM assembly using translation stages in each plane. The XBPM data for ID02 with a fix gap of $21.6 \mathrm{~mm}$ is shown in Fig. 7, when translation stages were scanned from $-1 \mathrm{~mm}$ to $1 \mathrm{~mm}$.

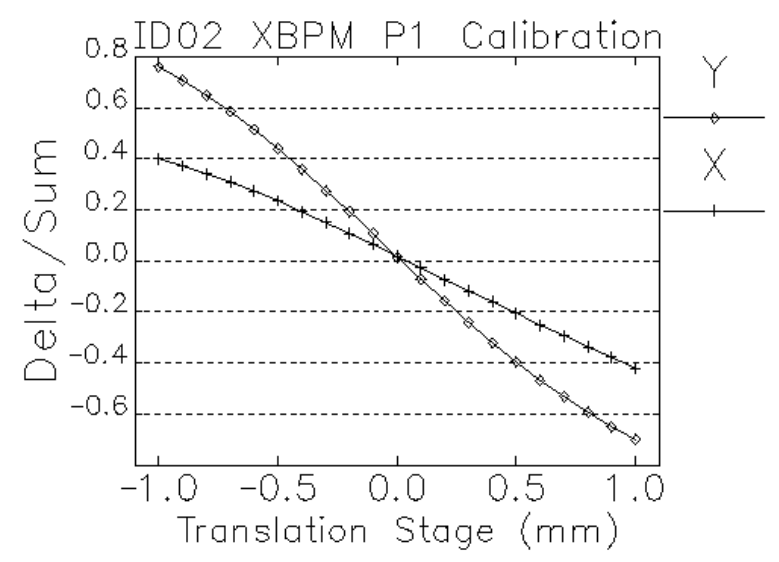

Figure 7: XBPM sensitivity (delta/sum) with translation stage scan.

The data were fit to a four-term polynomial:

$$
\begin{aligned}
& Y=+0.018-0.870 \mathrm{~m}+0.015 \mathrm{~m}^{2}+0.143 \mathrm{~m}^{3} \\
& X=-0.020-0.449 \mathrm{~m}-0.028 \mathrm{~m}^{2}+0.039 \mathrm{~m}^{3}
\end{aligned}
$$

where $\mathrm{m}$ represents translation stage motion in $\mathrm{mm}$. In the linear range,

$$
\begin{aligned}
& \mathrm{K} 1_{\mathrm{y}}=1 / 0.870=1.149 \\
& \mathrm{~K} 1_{\mathrm{x}}=1 / 0.449=2.227
\end{aligned}
$$

These gain factors were inserted in XBPM electronics. The second step is to generate local bumps using four correctors and two RFBPMs, straddling the ID. For simplification, two experiments were done, each scanning one RFBPM at a time. The scan setup diagram is shown in Fig. 8. In the top setup (first experiment), the downstream RFBPM (AP0) is scanned, while keeping the upstream RFBPM (BP0) fixed. This setup provides calibration for the downstream RFBPM. The bottom set up (second experiment) is used for the upstream RFBPM calibration.

Let us say that a, b, and c are the distances of BP0, $\mathrm{P} 1$, and $\mathrm{P} 2$ from AP0, as shown in Fig. 8. The two calibration values for each AP0 and BP0 are then calculated as: 
First experiment:

$$
\begin{gathered}
(\mathrm{AP} 0)=[(\mathrm{a}) /(\mathrm{a}+\mathrm{b})](\mathrm{P} 1), \text { and } \\
(\mathrm{AP} 0)=[(\mathrm{a}) /(\mathrm{a}+\mathrm{c})](\mathrm{P} 2)
\end{gathered}
$$

Second experiment:

$$
\begin{gathered}
(\mathrm{BP} 0)=(\mathrm{a} / \mathrm{b})(\mathrm{P} 1), \text { and } \\
(\mathrm{BP} 0)=(\mathrm{a} / \mathrm{c})(\mathrm{P} 2)
\end{gathered}
$$

The same equation is used for the horizontal as well as the vertical plane. The ratio of the above-calculated values to the directly measured $\mathrm{P} 0$ values is computed and is shown in Table 1. The average of two calibration values is shown in the third row. The average values are within $10 \%$, which is quite good.

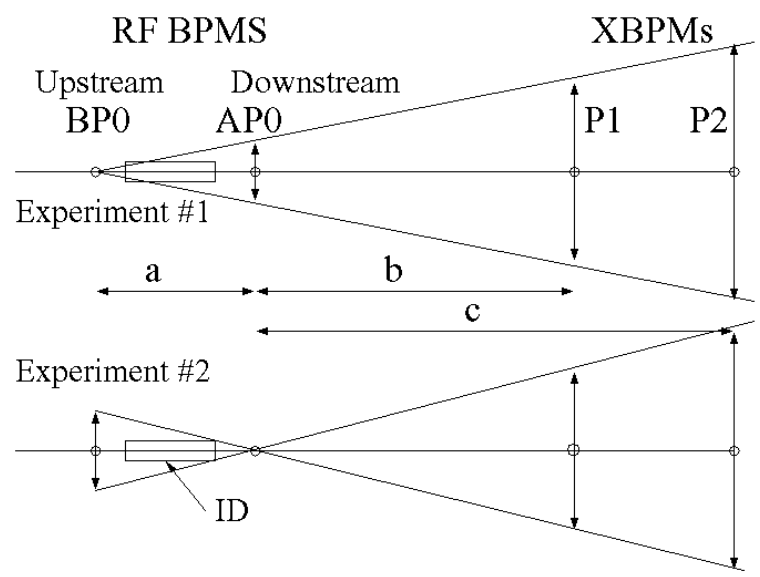

Figure 8: Calibration setup for RFBPMs straddling ID.

Table 1: rf BPM Calibration Data

\begin{tabular}{|c|c|c|c|c|}
\hline $\begin{array}{c}\text { XBPM \# } \\
\text { No. }\end{array}$ & $\begin{array}{c}\text { RFBPM } \\
\text { BP0-x }\end{array}$ & $\begin{array}{c}\text { RFBPM } \\
\text { BP0-y }\end{array}$ & $\begin{array}{c}\text { RFBPM } \\
\text { AP0-x }\end{array}$ & $\begin{array}{c}\text { RFBPM } \\
\text { AP0-y }\end{array}$ \\
\hline XBPM-P1 & 1.054 & 1.006 & 1.043 & 1.024 \\
\hline XBPM-P2 & 1.131 & 0.952 & 1.012 & 0.992 \\
\hline Average & 1.092 & 0.979 & 1.028 & 1.008 \\
\hline
\end{tabular}

\section{SUMMARY}

The implementation of new DSP-based data acquisition and control hardware has allowed a smooth integration of XBPM data with existing storage ring RFBPM data into a unified orbit control algorithm. XBPMs from several BM beamlines have been used in orbit feedback during operation routinely, with remaining units to be implemented by the end of this year. For the ID beamlines, lattice modification has been performed in about 10 sectors, with more to follow. The orbit feedback has been tested at a fixed gap. Work continues to provide reliable operation with variable gaps. XBPM translation stages have been employed for calibrating RFBPMs.

\section{ACKNOWLEDGMENTS}

We wish to thank M. Borland, J. Carwardine, L. Emery, L Erwin, S.Farrell, H. Friedsam, C. Gold, M.Hahne, R. Laird, F. Lenkszuz, A. Pietryla, M. Ramanathan, T. Roberts, S. Sharma, and D. Shu for their contributions.

\section{REFERENCES}

[1] D. Shu, B. Rodricks, J. Barraza, T. Sanchez, and T. M. Kuzay, "The APS x-ray undulator photon beam position monitor and tests at CHESS and NSLS," Nucl. Instrum. Methods Phys. Res., Sect. A 319, 56 (1992).

[2] O. Singh, L. Erwin, G. Decker, R. Laird, F. Lenkszus, "X-ray BPM-based feedback system at the APS storage ring," BIW 2000, Cambridge, MA (May 8-11, 2000).

[3] F. Lenkszus, R. Laird, G. Decker, O. Singh, M. Hahne, and L. Erwin, "New x-ray BPM data acquisition system for APS" these proceedings (2001).

[4] G. Decker, "Orbit control at the Advanced Photon Source" DIPAC 2001, ESRF, Grenoble, France (May 13-15, 2001).

[5] G. Decker, "Strategy for achieving true submicron rms orbit stabilization at the APS," these proceedings (2001).

[6] L. Emery and M. Borland, "Advances in orbit drift correction in the Advanced Photon Source," Proc. 1997 PAC, IEEE 97CH36167, Vancouver BC, pp. $742-744$ (1997).

[7] J. Carwardine and F. Lenkszus, "Real-time orbit feedback at the APS," BIW 1998, AIP Conference Proceedings 451 (1998).

[8] T. Warwick, N. Andresen, G. Portmann, and A. Jackson, "Performance of photon position monitors and stability of undulator beamline at the advanced light sources," Rev. Sci. Instrum. 66 (2) (1995).

[9] K. Holldack, J. Feikes, and W.B. Peatman, BESSY "Review of emmittance and stability monitoring using synchrotron radiation monitors" DIPAC 2001, ESRF, Grenoble, France (May 1315, 2001).

[10] G. Decker and O. Singh, "Method of reducing xray background signals from insertion device $\mathrm{x}$ ray beam position monitors," Phys. Rev. St. Accel. Beams 2, 112801 (1999).

[11] G. Decker, O. Singh, H. Friedman, J. Jones, M. Ramanathan, and D. Shu, "Reduction of X-BPM systematic errors by modification of lattice in the APS storage ring" 1999 PAC, IEEE 99CH36366, New York (1999). 